\title{
A simple protocol for the subcellular fractionation of skeletal muscle cells and tissue
}

\author{
Ivan Dimauro ${ }^{2}$, Timothy Pearson ${ }^{1}$, Daniela Caporossi ${ }^{2}$ and Malcolm J Jackson ${ }^{1 *}$
}

\begin{abstract}
Background: We describe a method for subcellular fractionation of mouse skeletal muscle, myoblast and myotubes to obtain relatively pure fractions of nuclear, cytosolic and mitochondrial compartments. Fractionation allows the analysis of a protein of interest (or other cellular component) based on its subcellular compartmental distribution and can also generate molecular information about the state of a cell and/or tissue and how the distribution of a protein may differ between different cellular compartments, tissues or cell types, in response to treatments or ageing.

Findings: The described method was specifically developed for skeletal muscle and proliferating/differentiated muscle cells. The purity of the different fractions, representing the cytoplasmic, mitochondrial and nuclear subcellular compartments was validated by western blot analysis of "house-keeper" marker proteins specific for each cellular compartment.

Conclusion: This low cost method allowed the mitochondrial, cytoplasmic and nuclear subcellular compartments from the same starting muscle samples to be rapidly and simultaneously isolated with good purity and without the use of an ultracentrifuge. This method permits samples to be frozen at $-80^{\circ} \mathrm{C}$ for future analysis and/or additional processing at a later date.
\end{abstract}

Keywords: Skeletal muscle, Subcellular fractionation, Western blotting

\section{Findings}

\section{Background}

Isolation of nuclear, cytosolic and mitochondrial fractions of reasonable purity from mammalian tissues and cells has generated great interest as it has the advantage of allowing different cellular proteins and organelles to be studied and characterised. Subcellular fractionation is universally used for various cell types and tissues for sample preparation and prior to subsequent $\sim$ omics analysis [1-5]. Generic fractionation protocols exist that can purify specific subcellular compartments and organelles, but in general they are not tailored for use with skeletal muscle and may require large amounts of starting material, time, or special reagents whilst potentially yielding fewer fractions from the same starting sample etc. [3,5-9]. The protocol described has been optimized for use with primary skeletal

\footnotetext{
*Correspondence: mij@liverpool.ac.uk

'Department of Musculoskeletal Biology, Institute of Ageing \& Chronic Disease, University of Liverpool, Daulby Street, Liverpool L69 3GA, United Kingdom

Full list of author information is available at the end of the article
}

muscle tissue (e.g. mouse anterior tibialis (AT) muscle) and both proliferating and differentiated $\mathrm{C} 2 \mathrm{C} 12$ cells to isolate subcellular fractions of nuclei, cytosol, and mitochondria from a single starting sample, thereby reducing the quantity of starting material, cost and total time needed for sample preparation.

The protocol works well for skeletal muscle tissue and cells and could be used as a starting point for the fractionation of other non-muscle samples although changes to buffer volumes; homogenization duration/intensity etc. may be required. The purity of the fractions obtained was assessed by immunoblotting for specific protein markers: histone H3 (nuclei), glyceraldehyde 3-phosphate dehydrogenase (GAPDH, cytosol), and cytochrome oxidase IV (CoxIV, mitochondria).

\section{Cell culture and animals}

The $\mathrm{C} 2 \mathrm{C} 12$ mouse skeletal myoblast cell line was obtained from the American Type Culture Collection (CRL-1772). C2C12 myoblasts were maintained in DMEM (Sigma Aldrich, Poole, UK) supplemented with 
1\% L-glutamine (Lonza, Cologne, Germany), 10\% FBS (Biosera, Sussex, UK) and $1 \%$ penicillin and streptomycin (Sigma) under an atmosphere of $5 \% \mathrm{CO}_{2}$ in humidified air at $37^{\circ} \mathrm{C}$. To induce myogenic differentiation, the growth medium was changed to differentiation medium (DMEM supplemented with $2 \%$ horse serum (Sigma) and 1\% antibiotics) after myoblasts had reached $\approx 90 \%$ confluence in a T75 $\mathrm{cm}^{2}$ flask. Myoblast cells were either harvested at $90 \%$ confluence or allowed to mature to myotubes for 7 days and then harvested (see below).

Adult mice (C57BL/6) were euthanized by overdose with anesthetic (ketamine hydrochloride and medatomidine hydrochloride) administered by intraperitoneal injection. Anterior tibialis (AT) muscles, approximately $50 \mathrm{mg}$ wet weight, were rapidly removed and used fresh to prepare fractions. Experiments were performed in accordance with UK Home Office Guidelines under the UK Animals (Scientific Procedures) Act 1986 and received ethical approval from the University of Liverpool Animal Welfare Committee.

\section{Subcellular fractionation}

Fresh AT tissue and scraped cells were washed with cold PBS, cells were pelleted by centrifugation at $200 \mathrm{~g}$ for 7 minutes whereas tissues were placed in a pre-chilled glass Petri dish and minced on ice using sharp scissors. All samples were resuspended in 300-500 $\mu \mathrm{l}$ of STM buffer comprising $250 \mathrm{mM}$ sucrose, $50 \mathrm{mM}$ Tris- $\mathrm{HCl}$ $\mathrm{pH}$ 7.4, $5 \mathrm{mM} \mathrm{MgCl}_{2}$, protease and phosphatase inhibitor cocktails (all chemicals were from Sigma-Aldrich, Poole, UK unless stated otherwise) and homogenized for 1 minute on ice using a tight-fitting Teflon pestle attached to a Potter S homogeniser (Sartorius Stedium, Goettingen, Germany) set to 600-1,000 rpm. The homogenate was then inspected, if intact tissue was still evident the homogenisation was repeated. The homogenate was decanted into a centrifuge tube and maintained on ice for 30 minutes, vortexed at maximum speed for 15 seconds and then centrifuged at $800 \mathrm{~g}$ for 15 minutes. The pellet was labelled as $\mathrm{P}_{0}$ and kept on ice, the supernatant was labelled as $S_{0}$ and used for subsequent isolation of mitochondrial and cytosolic (Figure 1) fractions.

The pellet $\mathrm{P}_{0}$ (containing nuclei and debris) was resuspended in 300-500 $\mu$ l STM buffer, vortexed at maximum speed for 15 seconds and then centrifuged at $500 \mathrm{~g}$ for 15 minutes. Following the above step, the nuclear pellet was labelled as $P_{1}$ and kept on ice, the supernatant $S_{1}$ (cell debris) was discarded. The purity of the nuclei within fraction P1 can be quickly determined by microscopic inspection by diluting an aliquot of the fraction in a trypan blue solution on a haemocytometer. If the $\mathrm{P}_{1}$ fraction contained excess cell debris the above step was repeated once.
To increase the $\mathrm{P}_{1}$ fraction purity further it was washed in STM buffer (300-500 $\mu \mathrm{l})$, vortexed at maximum speed for 15 seconds and then centrifuged at $1,000 \mathrm{~g}$ for 15 minutes. The washed pellet was labelled as $\mathrm{P}_{5}\left(\mathrm{~S}_{5}\right.$ was discarded $)$ and resuspended in 200-500 $\mu \mathrm{l}$ NET buffer (comprising: $20 \mathrm{mM}$ HEPES pH 7.9, $1.5 \mathrm{mM}$ $\mathrm{MgCl}_{2}, 0.5 \mathrm{M} \mathrm{NaCl}, 0.2 \mathrm{mM}$ EDTA, 20\% glycerol, $1 \%$ Triton-X-100, protease and phosphatase inhibitors) using a pipette to triturate until homogeneous. Pellet $\mathrm{P}_{5}$ was vortexed at maximum speed for 15 seconds and incubated on ice for 30 minutes, this fraction contained the nuclei. The nuclei were lysed with $10-20$ passages through an 18-gauge needle and/or sonicated (using a Soniprep 150, MSE, London, UK) at high setting for 1015 seconds with 30 second pauses whilst being kept on ice throughout. The lysate was centrifuged at 9,000 $\mathrm{g}$ for 30 minutes (at $4^{\circ} \mathrm{C}$ ), the resultant supernatant $\left(\mathrm{S}_{6}\right)$ was the final "nuclear fraction" (Figure 1).

Cytosolic and mitochondrial fractions were extracted from $\mathrm{S}_{0}$ by centrifugation at $800 \mathrm{~g}$ for 10 minutes. The supernatant $\mathrm{S}_{2}$ was saved and the pellet $\left(\mathrm{P}_{2}\right)$ was discarded, though to improve the nuclear yield the pellet $\mathrm{P}_{2}$ can be combined with fraction $\mathrm{P}_{0}$ (optional step). $\mathrm{S}_{2}$ was then centrifuged at $11,000 \mathrm{~g}$ for 10 minutes and the supernatant $\mathrm{S}_{3}$ (containing cytosol and microsomal fraction) was precipitated in cold $100 \%$ acetone at $-20^{\circ} \mathrm{C}$ for at least 1 hour followed by centrifugation at $12,000 \mathrm{~g}$ for 5 minutes, the pellet $\left(\mathrm{P}_{7}\right)$ was then resuspended in 100$300 \mu \mathrm{l}$ STM buffer and labelled as "cytosolic fraction" (Figure 1) that likely included some microsomal content. The pellet $\mathrm{P}_{3}$ was again resuspend in 100-200 $\mu$ STM buffer and centrifuged at $11,000 \mathrm{~g}$ for 10 minutes. Once centrifuged, supernatant $\mathrm{S}_{4}$ was discarded, the mitochondrial pellet $\left(\mathrm{P}_{4}\right)$ was resuspended in 50-100 $\mu$ SOL buffer (comprising: $50 \mathrm{mM}$ Tris $\mathrm{HCl} \mathrm{pH} \mathrm{6.8,} 1 \mathrm{mM}$ EDTA, 0.5\% Triton-X-100, protease and phosphatase inhibitors) by sonication on ice at high setting for 5-10 seconds with 30 second pauses and labelled as "mitochondrial fraction". All buffers and centrifugation steps were modified from Cox and Emili [9] and Psarra et al. [10].

Additionally, a commercial cell fractionation kit designed to yield near pure nuclei and cytoplasmic fractions from the same starting sample of cells and soft tissues was used (Thermo NE-PER nuclear and cytoplasmic extraction kit, Pierce-Thermo, Northumberland, UK, to obtain a mitochondrial fraction required the use of an additional kit) as directed by the manufacturer. This enabled a comparison of the efficacy of the commercial kit with the method described here when both methods were used to fractionate an identical starting sample (myoblast cells from a T75 $\mathrm{cm}^{2}$ flask, approximately $2 \times 10^{6}$ cells).

The protein content of each compartment was determined using BCA protein assay (Sigma). 


\section{Protein fractions (nucleus, cytosol and mitochondria)}

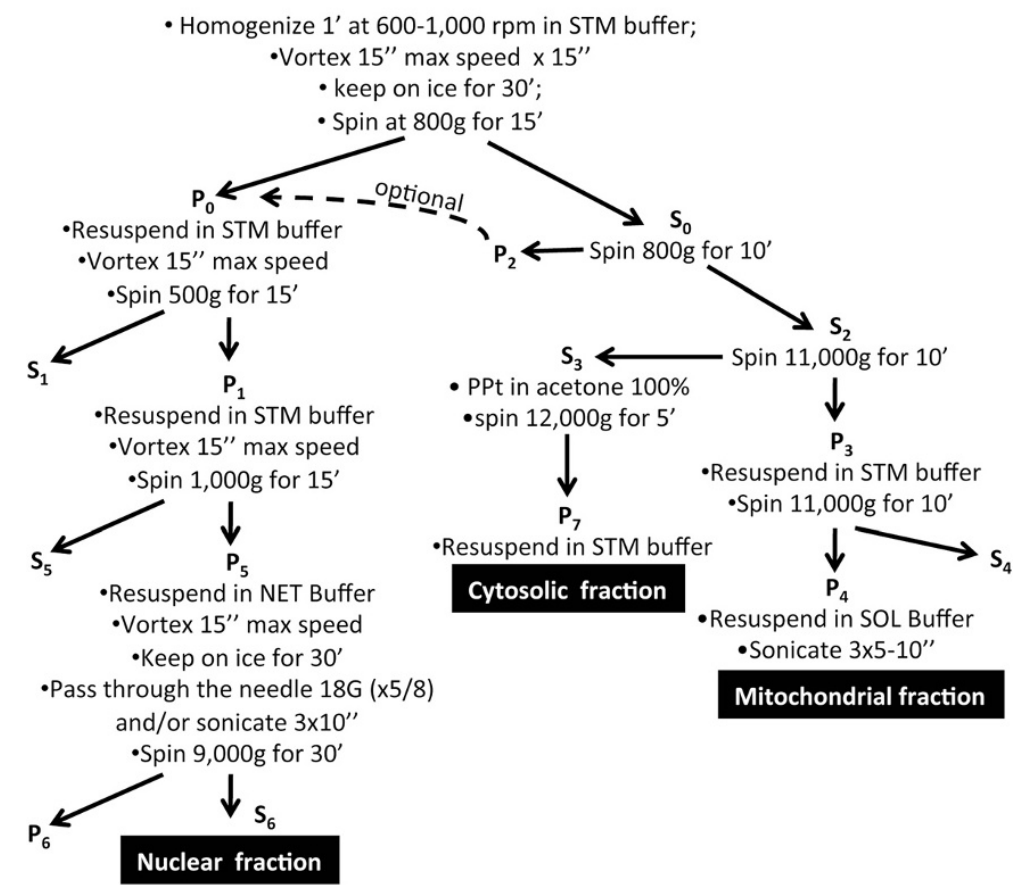

Figure 1 Schematic representation of the fractionation protocol. The developed protocol provides three subcellular fractions of cytoplasm, nuclei and mitochondria from a muscle sample. (- - ) Dotted arrow shows an optional step.

\section{Fractionation validation using western blotting}

Validation of the purity of the subcellular fractions derived from the same starting sample was determined by examining "house-keeper" (HK) protein markers by standard SDS-PAGE analysis. Protein samples (15-20 $\mu \mathrm{g} /$ fraction) were separated by a $12 \% \mathrm{SDS} /$ PAGE and transferred onto a nitrocellulose membrane (Sigma) and probed using monoclonal antibodies for Histone H3 (Cell Signalling, Hertfordshire, UK, 1: 2,000), GAPDH (Abcam, Cambridge, UK, 1: 5,000), and Cox IV (Abcam, Cambridge, UK, 1: 2,000) for nuclei, cytosolic and mitochondrial $\mathrm{HK}$ fractions respectively. Proteins were then visualized after applying specific secondary HRP-conjugated antibodies and exposure to a supersignal west dura substrate (PierceThermo, Northumberland, UK) by use of a ChemiDoc $^{\text {TM }}$ XRS (Bio-Rad, Hertfordshire, UK) see Figure 2 (representative example of at least three replicates per sample). Secondary antibody controls were also investigated and no non-specific binding was apparent (data not shown). To assess the purity of each fraction, bands relative to sub-compartment protein marker were quantified by ImageJ software [11].

\section{Results and discussion}

After fractionation and extraction of proteins from the $\mathrm{C} 2 \mathrm{C} 12$ cell cultures and AT muscles using the protocol described in Figure 1, we report the mean fraction yield values obtained from replicate experiments $(n=5)$ (Table 1). Figure 2A-C show the nuclear, cytoplasmic and mitochondrial fractions obtained from a single starting sample from each of the different tissue/cell types. The fractions were examined by western blotting analysis using antibodies directed against specific HK markers and revealed single dense bands for histone $\mathrm{H} 3$ at approximately $17 \mathrm{kDa}$ in the nuclear fraction, a band representing $\mathrm{GAPDH}$ at approximately $37 \mathrm{kDa}$ in the cytosolic fraction, and a single band at approximately $17 \mathrm{kDa}$ for CoxIV in the mitochondrial fraction (Figure 2A-C) in each sample.

These analyses showed little contamination between compartment fractions indicating that the purities of the nuclear, cytosolic, and mitochondrial fractions were relatively high (Table 2). The HK band intensity was high arguing that enrichment of proteins was achieved during processing (Table 1). The nuclear and mitochondrial fractions showed the highest band density indicating good yields and the lack of equivalent molecular weight bands in other fractions argued relatively little cross-talk and indicated reasonable purity, with the myotubes being slightly more variable depending on the fraction (feint additional non-specific bands were evident in the cytosolic fraction from myotubes). Furthermore, analysis of the distribution of the sub-compartment fraction 


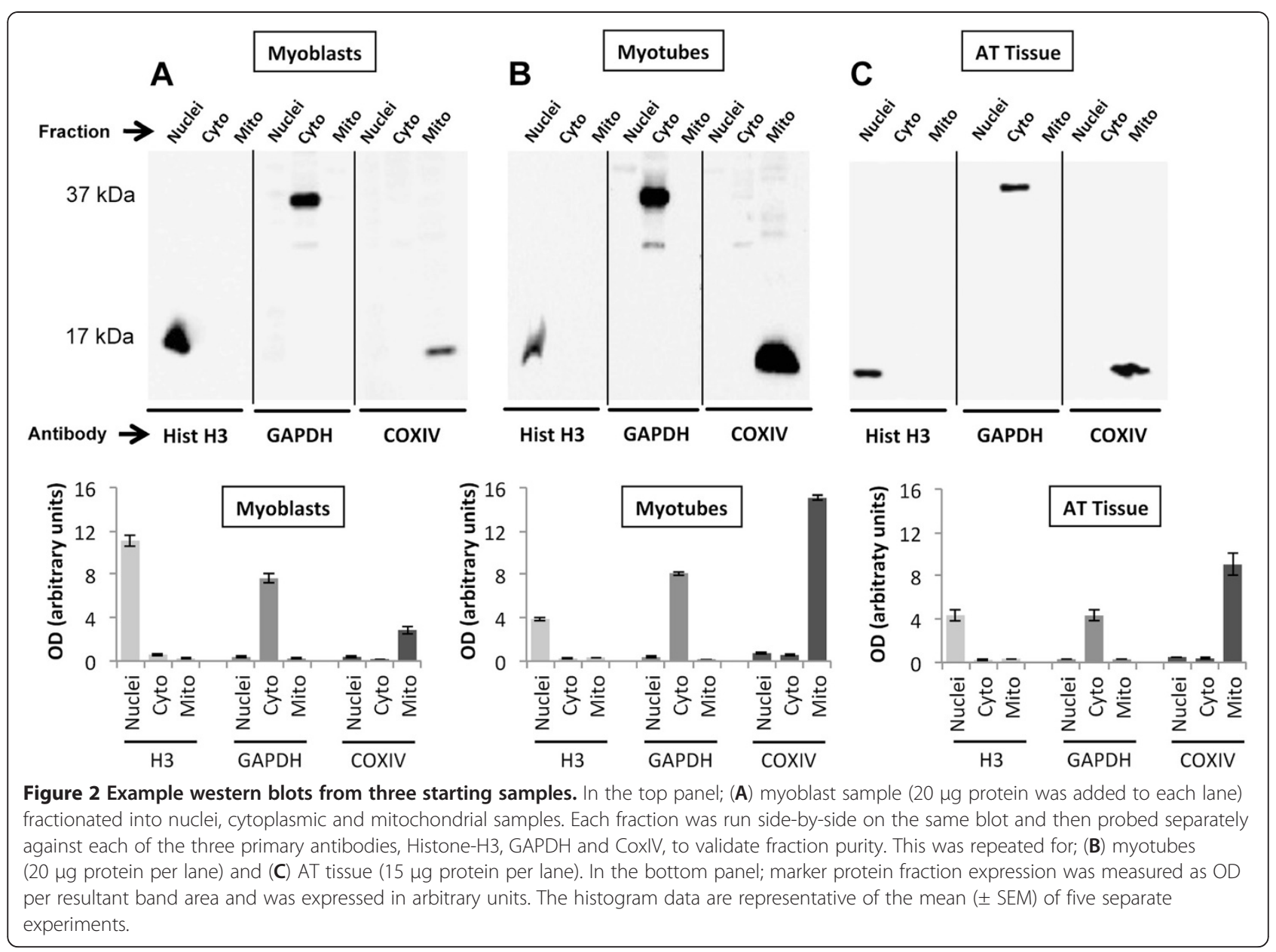

marker proteins (Table 2) showed the minimum percentage of purity was $\approx 85.6 \%$ (Cox IV mitochondrial marker in myoblasts) and therefore argued less than $15 \%$ was from other sources.

Comparison of the method described with a commercially available fractionation kit showed both approaches generated clean and abundant cytoplasmic fractions (Figure 3A), although the yield using the kit was smaller. The nuclear fraction generated by the kit showed both reduced yield and compromised purity (Figure $3 \mathrm{~B}$ ). The kit was not designed to generate a mitochondrial fraction and so no equivalent comparison was undertaken.

\section{Conclusion}

In comparison with the described protocol, the yield obtained with the commercial kit as indicated by band density, was poor and only the cytoplasmic fraction appeared relatively pure. The kit is designed for more generic use with multiple cells and soft tissues and may explain the inability to generate a pure and abundant nuclear fraction from muscle. The main advantage of

Table 1 Fraction yields for myoblast, myotube and AT tissue

\begin{tabular}{|c|c|c|c|c|c|}
\hline \multirow[t]{2}{*}{ Sample } & \multicolumn{3}{|c|}{ Mean protein yield $(\mu \mathrm{g})$} & \multirow{2}{*}{$\begin{array}{l}\text { Total sample } \\
\text { protein }(\mathrm{mg})\end{array}$} & \multirow[t]{2}{*}{ No. of replicates } \\
\hline & Nuclei & Cyto & Mito & & \\
\hline Myoblast & $1179 \pm 100(150 \mu \mathrm{L})$ & $496 \pm 25(100 \mu \mathrm{L})$ & $97 \pm 13(50 \mu \mathrm{L})$ & $1.8 \pm 0.1$ & 5 \\
\hline Myotube & $1624 \pm 107(150 \mu \mathrm{L})$ & $547 \pm 4(100 \mu \mathrm{L})$ & $197 \pm 15(50 \mu \mathrm{L})$ & $2.4 \pm 0.2$ & 5 \\
\hline AT tissue & $3294 \pm 254(300 \mu \mathrm{L})$ & $1486 \pm 225(300 \mu \mathrm{L})$ & $444 \pm 60(100 \mu \mathrm{L})$ & $5.2 \pm 0.5$ & 5 \\
\hline
\end{tabular}

Typical protein yield $(\mu \mathrm{g})$ obtained after sample fractionation using the indicated volume of lysis buffer for myoblasts, myotubes (both cultured in $75 \mathrm{~cm}^{2}$ flask, $\approx 2 \times 10^{6}$ cells) and AT muscle (all $n=5$, mean \pm SEM). The protein yields (determined by BCA assay as total volume of a fraction $\times$ protein concentration in the fraction) are representative and dependent upon the volume of buffer utilized to resuspend each fraction. 
Table 2 Purity analysis of the yield of sub-compartmental marker proteins

\begin{tabular}{llll}
\hline Fraction/Marker & Myoblasts & Myotubes & AT Tissue \\
\hline Nuclei/Histone H3 (\%) & $92.7 \pm 1.3$ & $86.4 \pm 1.4$ & $88.0 \pm 0.9$ \\
Cyto/GAPDH (\%) & $92.4 \pm 0.5$ & $93.9 \pm 1.4$ & $87.8 \pm 0.3$ \\
Mito/CoxIV (\%) & $85.6 \pm 0.9$ & $92.1 \pm 0.5$ & $91.2 \pm 0.1$
\end{tabular}

The percentage shown in the table is obtained by the ratio between OD values of Histone H3, GAPDH and CoxIV (all $n=5$, mean \pm SEM) in an individual target compartment divided by the sum of the $\mathrm{OD}$ values present in all compartments in each sample.

the kit was a slightly reduced processing time although an additional kit to purify a mitochondrial (and cytoplasmic) fraction would be required and this would increase the cost and time requirement.

In conclusion the method for subcellular fractionation described here is inexpensive, does not require an ultracentrifuge and was found to generate three relatively abundant subcellular fractions of reasonable purity. This method of subcellular fractionation could be combined with proteomics research wherein protein patterns of subcellular fractions could be mapped and characterized by $2 \mathrm{D}$ gel analysis and mass spectrometry.

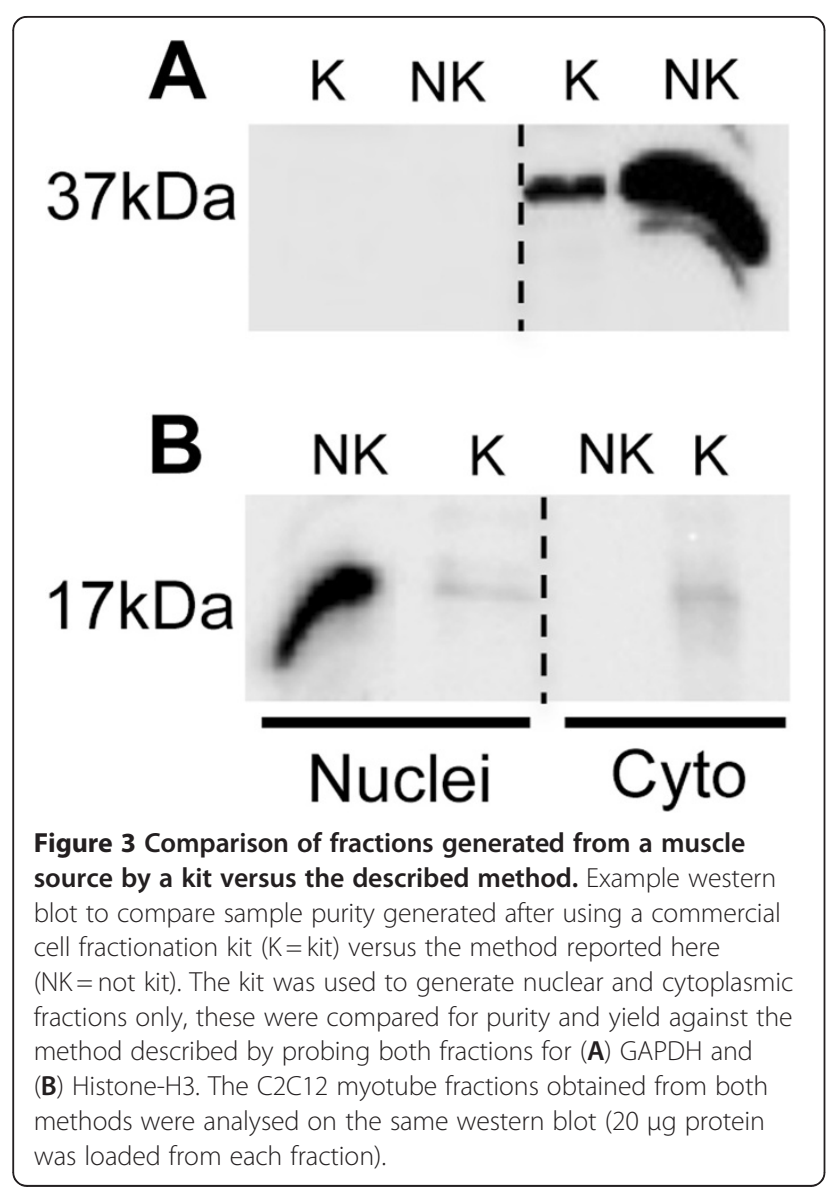

Competing interests

The authors declare that they have no competing interests.

\section{Authors' contributions}

ID and TP designed and carried out the cell culture and tissue experiments; MJJ and TP developed and supervised the experiments; MJJ, ID, TP and DC interpreted the data and wrote the manuscript. All authors read and approved the final manuscript.

\section{Authors' information}

Ivan Dimauro and Timothy Pearson joint first author.

\section{Acknowledgements}

Funding by the MRC (TP, MJJ) and University of Rome "Foro Italico" (ID, DC) are gratefully acknowledged.

\section{Author details}

${ }^{1}$ Department of Musculoskeletal Biology, Institute of Ageing \& Chronic Disease, University of Liverpool, Daulby Street, Liverpool L69 3GA, United Kingdom. 'Department of Health Sciences, University of Rome "Foro Italico", Piazza Lauro De Bosis 15, 00194, Rome, Italy.

Received: 5 April 2012 Accepted: 31 August 2012

Published: 20 September 2012

\section{References}

1. De Duve C, Pressman BC, Gianetto R, Wattiaux R, Appelmans F: Tissue fractionation studies. 6. Intracellular distribution patterns of enzymes in rat-liver tissue. Biochem J 1955, 60:604-617.

2. Bronfman M, Loyola G, Koenig CS: Isolation of intact organelles by differential centrifugation of digitonin-treated hepatocytes using a table Eppendorf centrifuge. Anal Biochem 1998, 255:252-256.

3. Srinivas KS, Chandrasekar G, Srivastava R, Puvanakrishnan R: A novel protocol for the subcellular fractionation of C3A hepatoma cells using sucrose density gradient centrifugation. J Biochem Biophys Methods. 2004, 60:23-27.

4. Kislinger T, Cox B, Kannan A, Chung C, Hu P, Ignatchenko A, Scott MS, Gramolini AO, Morris Q, Hallett MT, Rossant J, Hughes TR, Frey B, Emili A: Global survey of organ and organelle protein expression in mouse: combined proteomic and transcriptomic profiling. Cell 2006, 125:173-186.

5. Holden P, Horton WA: Crude subcellular fractionation of cultured mammalian cell lines. BMC Res Notes. 2009, 2:243.

6. Mootha VK, Bunkenborg J, Olsen JV, Hjerrild M, Wisniewski JR, Stahl E, Bolouri MS, Ray HN, Sihag S, Kamal M, Patterson N, Lander ES, Mann M: Integrated analysis of protein composition, tissue diversity, and gene regulation in mouse mitochondria. Cell 2003, 115:629-640.

7. Krapfenbauer K, Fountoulakis M, Lubec G: A rat brain protein expression map including cytosolic and enriched mitochondrial and microsomal fractions. Electrophoresis 2003, 24:1847-1870.

8. Dreger M, Bengtsson L, Schöneberg T, Otto H, Hucho F: Nuclear envelope proteomics: novel integral membrane proteins of the inner nuclear membrane. Proc Natl Acad Sci U S A 2001, 98:11943-11948.

9. Cox B, Emili A: Tissue subcellular fractionation and protein extraction for use in mass-spectrometry-based proteomics. Nature Protocols 2006, 4:1872-1878.

10. Psarra AM, Solakidi S, Trougakos I, Margaritis L, Spyrou G, Sekeris C: Glucocorticoid receptor isoforms in human hepatocarcinoma HepG2 and SaOS-2 osteosarcoma cells: Presence of gluocorticoid receptor alpha in mitochondria and of glucocorticoid receptor beta in nucleoli. The Int. Jnl. Biochem. \& Cell Biol 2005, 37:2544-2558.

11. Rasband WS, Image J: US National Institutes of Health. Maryland, USA: Bethesda; 1997-2008. http://rsb.info.nih.gov/ij.

\section{doi:10.1186/1756-0500-5-513}

Cite this article as: Dimauro et al: A simple protocol for the subcellular fractionation of skeletal muscle cells and tissue. BMC Research Notes 2012 5:513. 